\title{
O tratamento cirúrgico é imperativo na lesão do ligamento cruzado anterior? Há lugar para o tratamento conservador?
}

\author{
Is surgical treatment mandatory for anterior cruciate ligament \\ lesions? Can conservative treatment be considered?
}

\author{
Marco Martins Amatuzzi ${ }^{1}$, Roberto Freire da Mota e AlbuQueroue ${ }^{2}$, \\ MARIA LUIZA AMATUZZI ${ }^{3}$, SANDRA UMEDA SASAKI ${ }^{3}$
}

\section{RESUMO}

$O$ presente trabalho visa responder às questões expostas no título. Desde 1980, a indicação cirúrgica no tratamento da lesão do ligamento cruzado anterior (LCA) é um consenso e, hoje, a disseminação da técnica artroscópica, uma realidade, inclusive nos meios acadêmicos. Por esse motivo, a terapêutica indicada para tratar a lesão ligamentar mais comum do joelho, a lesão do LCA, passou a ser a reconstrução isolada desse ligamento, deixando-se então de considerá-la dentro do conceito das instabilidades decorrentes do trauma rotacional. Foi realizada revisão de publicações sobre o tratamento da lesão do LCA: artigos históricos, artigos publicados na Revista Brasileira

* Trabalho realizado no Hospital das Clínicas da Faculdade de Medicina da Universidade de São Paulo - USP - São Paulo (SP), Brasil

1. Professor Emérito da Faculdade de Medicina da Universidade de São Paulo - USP - São Paulo (SP), Brasil.

2. Doutor, Médico Assistente do Instituto de Ortopedia e Traumatologia do Hospital das Clínicas da Faculdade de Medicina da Universidade de São Paulo - USP - São Paulo (SP), Brasil.

3. Doutora em Ciências, Departamento de Ortopedia e Traumatologia, Faculdade de Medicina da Universidade de São Paulo - USP - São Paulo (SP), Brasil.

Endereço para correspondência: Al. Itu, 112, apto. 81 - 01421-000 - São Paulo, SP.

Copyright RBO2007 de Ortopedia nos últimos sete anos e 13 artigos na literatura internacional, selecionados a partir da sua qualidade metodológica. Acrescentou-se um estudo de coorte da casuística da reconstrução do LCA dos próprios autores. $\mathrm{Na}$ maioria dos trabalhos nacionais revistos, a indicação de tratamento cirúrgico é peremptória e os detalhes clínicos da constatação dessa lesão, aguda e crônica, são dados, unicamente, pela positividade dos testes, da gaveta anterior, Lachman e jerk test ou pivot shif, sem a avaliação dos ligamentos periféricos ou a própria constatação do tipo de instabilidade e a quantificação dos seus componentes. Nos 13 trabalhos internacionais, observou-se que o tratamento conservador do LCA é uma opção considerada, principalmente pelos resultados apresentados por pacientes assim conduzidos. No estudo da casuística própria, verificou-se que, dentre os pacientes operados, um número significativo de joelhos (55), correspondente a $6,2 \%$ do total, apareceu com nova lesão antes do segundo ano; esses eram justamente aqueles que haviam sido operados somente do LCA, apesar de ter apresentado testes positivos de instabilidade rotacional de moderada magnitude. Pode afirmar-se, assim, que é possível tratar conservadoramente a lesão do LCA e, que, portanto, o tratamento cirúrgico não é imperativo e, ainda, que esse, quando indicado, deverá ser planejado, considerando-se o grau de frouxidão decorrente da lesão dos ligamentos periféricos.

Descritores - Ligamento cruzado anterior/cirurgia; Ligamento cruzado anterior/terapia; Artroscopia; Literatura de revisão 


\section{ABSTRACT}

The purpose of this paper is to answer the questions made in the title of the paper. Surgical indication for the treatment of anterior cruciate ligament (ACL) lesion has been a consensus since 1980, and today the widespread use of the arthroscopic technique is a reality, even in academic circles. For this reason, the therapy indicated to treat the most common ligament lesion of the knee - ACL lesion - has been the isolated reconstruction of the ligament, and the condition was no longer considered within the context of instabilities resulting from rotational trauma. A review of the literature dealing with ACL lesion treatment was carried out: historical papers, papers published in the Revista Brasileira de Ortopedia in the last seven years, and 13 papers from the international literature selected based on the quality of their method. A cohort study in the ACL reconstruction series of the authors was also included in the review. In most of the Brazilian papers reviewed, indication of surgical treatment is peremptory, and the clinical verification of the lesion, whether acute or chronic, is made just by the positive results of anterior drawer, Lachman, and jerk tests, or the pivot shift, without evaluating peripheral ligaments, the type of instability, and the quantification of components; in the 13 international papers, the authors observed that conservative ACL treatment is an option to be considered, in particular in view of the results presented by patients so treated. In their own series, the authors found that among patients operated on, a significant number of knees (55), corresponding to $6.2 \%$ of the total, presented a new lesion before the second year, and those were exactly the cases that had been operated on just for the ACL, in spite the positive, moderate magnitude results in rotational instability tests. A statement can be made that it is possible to treat an ACL lesion with conservative methods, and therefore surgical treatment is not mandatory, and, further, that when indicated, surgery must be planned considering the degree of ligament looseness resulting from the peripheral ligaments.

Keywords - Anterior cruciate ligament/surgery; Anterior cruciate ligament/therapy; Arthroscopy; Review literature
Hoje, um paciente com lesão do ligamento cruzado anterior (LCA), após adequadamente examinado e feito o diagnóstico, recebe uma proposta de tratamento cirúrgico. Esse é um conceito verdadeiro desde 1980, quando esses joelhos já eram operados, na fase aguda, com reconstrução intra-articular, associada ou não à extra-articular e, na fase crônica, através de técnicas extra-articulares inicialmente e, depois, com a reconstrução intra-articular, associada à extra-articular. Com a introdução do artroscópio, os joelhos passaram a ser tratados pela substituição isolada do LCA, programada, muitas vezes, sem levar em conta a situação de instabilidade rotatória quando é feito o dimensionamento de seus componentes.

Trabalhos científicos brasileiros, publicados nos últimos anos na Revista Brasileira de Ortopedia, reconhecem como boa essa técnica de reconstrução intra-articular, praticada como procedimento único. Esses trabalhos nacionais referem bons resultados, o que permite que a técnica cirúrgica de reconstrução isolada do LCA mantenha sua indicação ${ }^{(1-19)}$. No entanto, a observação mais atenta, nota-se que esses trabalhos são deficientes em seus desenhos e apresentam vieses, entre os quais a avaliação dos resultados feita a prazos variados ${ }^{(1,4,6-7,11,13-}$ 14,17,19), às vezes curtos demais, a não definição dos casos excluídos e as perdas de seguimento, entre outros vieses que invalidam as conclusões apresentadas.

O artroscópio é o instrumento preferido para execução dessas reconstruções ligamentares, na grande maioria das vezes. Método considerado pouco invasivo e de pouca morbidade, esbarra, no entanto, nas suas próprias limitações, entre as quais e talvez a mais importante, a de somente permitir procedimentos intra-articulares, onde a ótica e o instrumental são manobrados em meio líquido ou eventualmente gasoso; um procedimento extra-articular de reparação ou de reconstrução é praticamente impossível de ser feito com segurança, por via artroscópica.

A artroscopia chegou para tomar seu lugar nas operações sobre o joelho. As instituições de ensino passam a ensinar a técnica em laboratórios especiais, montados por grupos empresariais diretamente ligados ao marketing dos produtos ópticos. Nesses laboratórios aprendem-se o posicionamento do paciente, os portais, os ângulos de visibilidade e o adestramento manual necessário à efetivação da operação do equipamento, com o melhor aproveitamento e menor risco. A técnica torna-se essencial e razão de sobrevivência profissional. Os princípios biomecânicos, de difícil assimilação, através dos quais se compreendem a dinâmica dos movimentos da articulação central dos membros inferiores e a própria história natural da lesão do LCA, com sua sequiência que parte da perife- 
ria, não são estudados na sua profundidade. Não são consideradas as razões e motivos da indicação terapêutica, a seleção de pacientes para tratamento e o custo-benefício da intervenção. O ensinamento do básico é falho. O exame clínico do joelho não é detalhado, nem tampouco treinado; no entanto, são estudados e discutidos detalhes e minúcias dos exames complementares.

Em uma revisão sistemática dos trabalhos publicados sobre o assunto nos últimos seis anos, e até 2006, na Revista Brasileira de Ortopedia, e em teses de Ciência Básica, observa-se que poucos autores citam, no exame clínico do joelho com lesão ligamentar, os testes de avaliação das estruturas periféricas e seus respectivos valores ${ }^{(4-5)}$ e poucos ${ }^{(4-5,14,18-20)}$ mencionam os restritores secundários. Na grande maioria dos artigos é defendida a substituição isolada do LCA, independente do tipo de lesão detectada, como se todas elas fossem iguais. O tratamento cirúrgico é realizado, tendo como justificativa o retorno pleno à atividade física ou a profilaxia da artrose fatal ${ }^{(1-2,5-8,13-14,16,19-20)}$.

O método cirúrgico sobrepõe-se à teoria e à biomecânica; por ele deixa-se de lado a fisiopatologia.

Na literatura de ciência básica constata-se que a maioria dos trabalhos na área procura demonstrar as maneiras de fixar os enxertos ${ }^{(7,11-12,16)}$, os pontos ideais de inserção dos ligamentos centrais do joelho ${ }^{(17-18)}$, a realização de dois túneis ós$\operatorname{seos}^{(18,21-23)}$, a repercussão da retirada de enxerto de patela ${ }^{(19)}$, a frouxidão e o derrame decorrente da substituição do LCA ${ }^{(24)}$, o tensionamento ou pré-tensionamento do enxerto, etc., tudo com instrumental próprio de utilização.

$\mathrm{Na}$ discussão desse tema analisamos os resultados de um estudo de coorte dos pacientes por nós operados por instabilidades do joelho com lesão do LCA, agudos e crônicos. Grupo homogêneo de pacientes jovens, praticantes de esporte, cuja queixa principal era decorrente de trauma agudo, torcional do joelho ou instabilidade crônica, caracterizada principalmente por falseios. Do exame clínico desses pacientes constam todos os testes que detectam frouxidão ligamentar ${ }^{(5)}$, gaveta anterior nas três rotações do joelho, jerk test e manobras de abdução e adução em hiperextensão, 0 e $30^{\circ}$ de flexão do joelho, graduados nos seus componentes em leve $(+)$ moderado $(++)$ ou grave $(+++)$ e confirmados no exame sob anestesia, realizado na sala de operações. Em todos os pacientes foi realizada a reconstrução intra-articular usando-se a porção central do ligamento patelar, associada ou não à reconstrução extra-articular.

$\mathrm{Na}$ observação do quadro do estudo, verificamos que um número significativo de joelhos (55), correspondente a 6,2\% do total, apareceu com nova lesão antes do segundo ano; es- ses eram justamente aqueles que haviam sido operados somente do LCA, apesar de ter apresentado testes positivos de instabilidade rotacional de moderada magnitude, o que caracterizava lesão ou frouxidão periférica, não valorizada no planejamento cirúrgico (tabela 1 ).

TABELA 1

Avaliação periódica dos joelhos operados por lesão do LCA

\begin{tabular}{|c|c|c|c|c|c|}
\hline \multirow[t]{2}{*}{ Tempo (anos) } & \multicolumn{2}{|c|}{ Bom } & \multicolumn{2}{|c|}{ Regular } & Mau \\
\hline & № & $\%$ & № & $\%$ & № $\quad \%$ \\
\hline 2 & 874 & $(94,2)$ & 8 & $(0,8)$ & $55(6,2)$ \\
\hline 3 & 839 & (100) & & & \\
\hline 5 & 650 & (92) & & & $50(8,0)^{* *}$ \\
\hline 10 & 551 & (97) & 17 & $(3,0)^{* *}$ & \\
\hline 15 & 218 & (91) & 20 & $(0,9) * *$ & $1(0,4)$ \\
\hline 20 & 40 & (80) & & $(14) * *$ & $3(6,0)$ \\
\hline 25 & 28 & (80) & & (20) & \\
\hline
\end{tabular}

** relacionados com meniscectomia

Se consultarmos a literatura internacional, confirmamos que, quando a lesão periférica é detectada no exame clínico, ela deve ser quantificada em seus componentes e, conforme o caso, o tratamento cirúrgico deverá ser planejado associandose à reconstrução intra-articular a reconstrução extra-articu$\operatorname{lar}{ }^{(5,25-32)}$.

Recentemente, 2007, Fu et al, em editorial do Clinical Orthopaedics, enfocam a necessidade de desenvolver dinamômetros capazes de fornecer informações sobre a instabilidade rotacional do joelho, pois "os testes de Lachman e a KT 1000 avaliam somente o deslocamento ântero-posterior, não fornecendo informação suficiente", e que esse seria o caminho que deve alavancar as pesquisas para melhora do resultado de tratamento das lesões do $\mathrm{LCA}^{(33)}$.

Se voltarmos ainda à literatura internacional de qualidade, veremos que a própria indicação de tratamento cirúrgico para todas as lesões do LCA, independentemente da técnica empregada, pode ser questionada. Os pacientes tratados cirurgicamente, vistos a curto e médio prazos, retornam ao esporte, mas essa seria a única terapêutica possível para que se atinja esse fim? Como se justificam os muitos pacientes com lesão do LCA que retornam à função plena sem operação? Qual o especialista que não tem casos como esses para contar? Qual de nós não se surpreendeu quando, ao atender um paciente, verifica no seu prontuário uma proposta antiga de operação sobre um LCA rompido, não realizada por decisão do paciente, que hoje está bem e praticando esporte? Se a resposta à pergunta inicial for negativa, a indicação do tratamento cirúrgico preferencial, para a lesão do LCA, pode ser contestada. 
Para responder a esse questionamento, selecionamos na literatura internacional e nos últimos oito anos, até 2007, 13 trabalhos de boa qualidade metodológica, que estudam as possibilidades de tratamento da lesão do LCA, custo-benefício das diversas maneiras de tratar e resultados. Esses trabalhos, coincidentemente, são oriundos de países que praticam a medicina socializada, com exceção dos últimos, provenientes de Serviços dos Estados Unidos da América.

Citaremos, por ordem cronológica, Casteleyn, que, no seu artigo "Gerenciamento da lesão do LCA: uma moda que estava ganhando ou uma evidência científica?", fez uma revisão de literatura e, somando a população estudada, em sete artigos, encontra 636 joelhos tratados conservadoramente e, em 48 artigos, 3.500 joelhos operados. Esse autor conclui que nos casos que foram tratados sem operação, a necessidade de reconstrução secundária foi baixa (6\%) e que o tratamento cirúrgico envolveu alta morbidade osteoartrítica ${ }^{(34)}$.

No mesmo ano, 1999, na Alemanha, Diekstall et al observaram os resultados em três anos, de 16 joelhos operados e de 110 não operados, o que lhes permitiu concluir que, após ter introduzido a propriocepção no tratamento conservador, passaram a indicar a reconstrução somente a pacientes de alto risco ou quando, durante o tratamento conservador, os falseios apareciam ${ }^{(35)}$.

Na Suécia, Renström, do Grupo do Hospital Karolinska, em trabalho intitulado "Lesão do LCA - ainda um enorme desafio", propõe o tratamento conservador, que somente deverá ser interrompido quando houver referência de instabilidade; os autores suecos referem o aumento de risco de lesão meniscal, mas afirmam que a literatura não apresenta certeza de que o tratamento cirúrgico amenize esse risco ${ }^{(36)}$.

Reer et al, da Universidade de Hamburg, aplicaram o tratamento conservador em 41 joelhos que, randomizados, foram divididos em dois grupos, um dos quais usou uma joelheira tipo brace, e concluem que o uso desse tipo de órtese favoreceu o tratamento ${ }^{(37)}$.

Em 2002, no Japão, Fujimoto et al analisaram intra-operatoriamente o valor do deslocamento anterior da tíbia durante a operação de reconstrução do LCA, antes da retirada dos restos ligamentares e depois desse passo, na operação artroscópica. Esses autores usam KT 1000 e o resultado mostrou aumento dos valores do sinal da gaveta após a retirada do tecido cicatricial, o que revela o poder desse tecido em reter, mesmo que parcialmente, o deslocamento anterior do joelho e também sua capacidade de cicatrizar ${ }^{(38)}$.

Na Alemanha, em 2003, Hinterwimmer et al publicaram metanálise comparando os trabalhos de tratamento cirúrgico das lesões do LCA com o conservador e concluem: "Embora a reconstrução esteja estabelecida como padrão ouro, não há bases científicas para afirmar que operar o LCA lesado traga vantagem"(39).

Recentemente, são vistos trabalhos dos Estados Unidos da América, entre os quais destacamos, em 2005, o de coorte retrospectivo no qual os autores Craig et al acompanham 48 indivíduos com lesão LCA, testados com artrômetro, na operação, antes e depois da retirada da cicatriz (restos) do LCA ${ }^{(40)}$. Esse estudo confirma os achados de Fujimoto et al, de que essa cicatriz seria o ligamento reconstruído de forma aberrante, mas funcional ${ }^{(38)}$.

Em 2005, em estudo elaborado e mantido atualizado na Biblioteca Cochrane, Linko et al publicaram revisão sistemática, cujo objetivo foi avaliar o efeito do tratamento cirúrgico quando comparado com o conservador, na ruptura do LCA, em adultos. Os autores introduziram o assunto referindo que a lesão do LCA é comum e leva os joelhos à instabilidade, que há bons resultados funcionais com tratamento conservador, que essa é a alternativa de escolha para alguns pacientes, e sugerem que seja melhorada a qualidade das pesquisas que deverão analisar a efetividade das medidas terapêuticas e as alterações degenerativas; e mais, que a reconstrução parece recompor a estabilidade nas atividades extenuantes, mas que nada existe sobre o desenvolvimento de artrite em relação a qualquer tratamento ${ }^{(41)}$.

Fitzgerard et al, da Escola de Saúde e Ciências da Reabilitação da Universidade de Pittsburg, adotam uma lista de critérios para determinar a escolha do tratamento da lesão do LCA. Essa lista demonstra ser útil, pois, dos 93 joelhos com lesão do LCA, foram selecionados 39 para tratamento conservador e, desses, 28 (72\%) retornaram ao esporte no mesmo nível e jamais tiveram lesão meniscal ou sintomas de instabilidade; os 11 restantes, por ter tido episódios de falseios, retornaram ao cirurgião para tratamento operatório ${ }^{(42)}$.

Recentemente, Strehl et al apresentam seus casos, discorrendo sobre o protocolo de indicação de tratamento conservador e afirmando que, nesses pacientes selecionados e que realizaram o tratamento até o final, uma terça parte obteve bons ou muito bons resultados ${ }^{(43)}$.

Em decorrência dessas considerações e respondendo à pergunta ou objetivo deste trabalho, podemos afirmar que é possível tratar conservadoramente a lesão do LCA e que, portanto, o tratamento cirúrgico não é imperativo e que, quando indicado, deverá ser planejado considerando-se o grau de frouxidão decorrente da lesão dos ligamentos periféricos. 


\section{CRÍTICA}

Concordo com a maneira como o assunto - lesão do ligamento cruzado anterior - foi abordado neste artigo de atualização. $\mathrm{O}$ texto reflete conceituação atual e prática do problema. No Brasil, o esporte mais praticado é o futebol, esporte de contacto, no desempenho do qual, movimentos rotacionais são freqüentes, atividade em que a regulamentação não permite o uso de joelheiras anti-rotatórias. Isso muito contribuiu para a tendência, por muito tempo vigente, entre nós, de operar quase todos os casos de lesão do LCA. Essa não é a opinião dominante nos países em que a medicina baseada em evidências é valorizada. Estudos mostraram que é possível que atletas pratiquem vários esportes, com alto proveito, na vigência de lesão completa isolada do LCA. Exemplos são o triatlo (natação, bicicleta e corrida no plano), o golfe, o remo, a musculação, o velejar e o caminhar, entre outros esportes. Evidente que esses atletas não devem ter lesões meniscais ou condrais associadas. Portanto, o que se deve operar é a instabilidade rotatória ântero-lateral, decorrente da insuficiência do LCA, e não a lesão isolada do LCA que não determine instabilidade rotatória. Isso não é só válido nos esportes, mas em outras atividades do diaa-dia, salvaguardados: idade do paciente, sexo, peso corporal, estados clínico geral, etc...

Outro aspecto interessante no artigo é o que valoriza o exame das estruturas periféricas laterais e mediais que ajudam a proteger a articulação do joelho, pois, principalmente em lesões crônicas, somos obrigados a fazer também os reforços extra-articulares.

Existem hoje trabalhos científicos que mostram que o paciente que aceita viver sem a prática de atividades torcionais, que apresenta musculatura competente, adquirida após fisioterapia adequada, e que não sofreu torções, não vai desenvolver artrose, bem ao contrário do que era apregoado, por muitos autores, no passado.

\section{LUIZ ROBERTO StIgleR MARCZYK}

Professor Titular de Ortopedia da Faculdade de Medicina da Universidade Federal do Rio Grande do Sul

\section{REFERÊNCIAS}

1. Carneiro Filho M, Navarro RD, Laurino CFS, Benbassat JR. Reconstrução do ligamento cruzado anterior com auto-enxerto de tendão patelar por via artroscópica. Rev Bras Ortop. 1999;34(3):169-78.

2. Camanho GL, Andrade MH. Estudo comparativo da reabilitação dos pacientes submetidos à reconstrução do ligamento cruzado anterior com enxertos do terço médio do tendão patelar e com os dos tendões dos músculos flexores mediais do joelho. Rev Bras Ortop. 1999;34(9/10):513-8.

3. Mello A Júnior W, Penteado PCF, Marchetto A, Fatarelli IFC, Rodrigues RL, Cerqueira PH. História das lesões meniscais na reconstrução do ligamento cruzado anterior. Rev Bras Ortop. 1999;34(11/12):569-74.

4. Balsini N, Sardinha CE, Balsini NE. Tendão patelar "versus" tendões duplos do semitendinoso e "gracilis" como enxerto autólogo na reconstrução do LCA no joelho. Rev Bras Ortop. 2000;35(5):157-64.

5. Amatuzzi MM. Antigos conceitos são modernos no tratamento das doenças ligamentares do joelho. Rev Bras Ortop. 2001;36(1/2):1-8.

6. Camanho GL, Viegas AC. Avaliação da reconstrução do ligamento cruzado anterior em pacientes com idade acima de 45 anos. Rev Bras Ortop. 2001;36(1/2):37-40.

7. Severino NR, Camargo OPA, Aihara T, Cury RPL, Oliveira VM, Nishihara C. Utilização do parafuso "Bone Mulch" na reconstrução do ligamento cruzado anterior com tendões dos músculos semitendinoso e grácil. Rev Bras Ortop. 2001;36(3):79-83.

8. Camargo OPA, Severino NR, Aihara T, Cury RPL, Oliveira VM. Resultado a médio prazo de reconstrução da lesão crônica do ligamento cruzado anterior com prótese de poliéster. Rev Bras Ortop. 2001;36(4):111-6.

9. Zekcer A, Carneiro ACI, Minervini S, Carneiro Filho M. TransFix ${ }^{\circledR}$ : um método de fixação femoral dos tendões flexores na reconstrução do LCA. Relato preliminar. Rev Bras Ortop. 2001;36(9):340-4.

10. Boschin LC, Schuck GF, Oliveira G, Schmiedt I, Schwartsmann CR. Artrotomia "versus" artroscopia: avaliação pós-operatória da reconstrução do ligamento cruzado anterior. Rev Bras Ortop. 2002;37(1/2):23-30.

11. Gali JC, Adad MAH, Mod MSB. Reconstrução do ligamento cruzado anterior com tendões flexores quádruplos e parafusos de interferência metálicos. Rev Bras Ortop. 2002;37(6):240-6.

12. Silva JLV, Tavares Filho GS, Namba MM, Pereira Filho FA, Barbosa MA, Albano M, et al. Estudo biomecânico, in vitro, em ovinos, da fixação femoral do tendão patelar na reconstrução do LCA: comparação entre parafusos metálicos de interferência e a fixação sob pressão com bloco ósseo cônico. Rev Bras Ortop. 2003;38(7):400-9.

13. Guimarães MV. Reconstrução artroscópica do ligamento cruzado anterior: estudo comparativo entre os enxertos autólogos de ligamento patelar e de tendão do quadríceps. Rev Bras Ortop. 2004;39(1/2):30-41.

14. Gomes JLE, Marczyk LR. Reconstrução dos ligamentos cruzados do joelho com o tendão duplo do semitendinoso. Rev Bras Ortop. 2004;39(4): 137-46.

15. Hernandez AJ, Amatuzzi MM, Albuquerque RFM, Laraya MHF, Rubin MLL, Martinelli Filho M. Estudo prospectivo controlado do tratamento das lesões isoladas do ligamento cruzado anterior com reconstrução e osteotomias concomitantes. Rev Bras Ortop. 2004;39(7):371-81.

16. Camanho GL, Camanho LF, Viegas AC. Reconstrução do ligamento cruzado anterior com tendões dos músculos flexores do joelho fixos com Endobutton. Rev Bras Ortop. 2003;38(6):329-36.

17. Cortelazo MJ, Cohen M, Mestriner LA, Carneiro Filho M. Reconstrução artroscópica do ligamento cruzado anterior com enxerto do tendão quadricipital: estudos das características dimensionais do tendão e da técnica cirúrgica. Nota preliminar. Rev Bras Ortop. 2002;37(6):247-55. 
18. Domit Filho M, Monte APC, Nagai M, Ribeiro MV, Maciel LGB, Ribas Filho JM, Malafaia O. Estudo do posicionamento do enxerto na substituição do ligamento cruzado anterior. Rev Bras Ortop. 2002;37(4):141-50.

19. Andrade MAP, Cenni MHF, Pinheiro Júnior LFB, Lemos WG. A repercussão da retirada do enxerto do tendão patelar no mecanismo extensor do joelho. Rev Bras Ortop. 1999;34(8):461-4.

20. Lustosa LP, Andrade MAP, Fonseca ST, Bittencourt NFN. Análise do nível de frouxidão ligamentar e do desempenho funcional em indivíduos submetidos à reconstrução do LCA com o uso do terço central do ligamento patelar. Rev Bras Ortop. 2006;41(3):70-5.

21. Albuquerque RF, Sasaki SU, Amatuzzi MM, Angelini FJ. Anterior cruciate ligament reconstruction with double bundle versus single bundle: experimental study. Clinics. 2007;62(3):335-44.

22. Kokron AEV. Avaliação biomecânica da estabilidade da reconstrução na lesão isolada do ligamento cruzado posterior com um e dois feixes de enxerto: estudo experimental com tendão do músculo quadríceps da coxa e com tendões dos músculos semitendíneo e grácil [tese]. São Paulo: Faculdade de Medicina da Universidade de São Paulo; 2000.

23. Sasaki SU. Estudo biomecânico comparativo, em cadáveres, da reconstrução do ligamento cruzado anterior do joelho com técnica convencional e com túneis duplos tibiais e femorais [tese]. São Paulo: Faculdade de Medicina da Universidade de São Paulo; 2007.

24. Carvalho Júnior LH, Denaro MF, Gonçalves TJ, Brito HMC, Gonçalves MJB. Uso do dreno de sucção após a reconstrução do ligamento cruzado anterior. Rev Bras Ortop. 2006;41(6):211-6.

25. Dejour H, Chambat P. Las laxitudes crónicas anteriores. In: Symposium Internacional de Traumatologia, 6 ${ }^{\circ}, 1980$, Madrid. Lesiones de la rodilla: generalidades, exploración, osteocondritis, fracturas de la rodilla, rotula, meniscos, ligamentos, artrosis y otras lesiones. Madrid: Fundación Mapfre; 1980. p. 721-9.

26. Clancy WG Jr, Nelson DA, Reider B, Narechania RG. Anterior cruciate ligament reconstruction using one-third of the patellar ligament, augmented by extra-articular tendon transfers. J Bone Joint Surg Am. 1982;64(3):352-9.

27. Puddu G, Hippolito E. Reconstruction of the anterior cruciate ligament using the semitendinosus tendon. Histological study of a case. Am J Sports Med. 1983;11(1):14-6.

28. Dejour H, Walch G, Neyret P, Adeleine P. Résultats des laxités chroniques antérieures opérées. A propos de 251 cas revus avec un recul minimum de 3 ans. Rev Chir Orthop Reparatrice Appar Mot. 1988;74(7):622-36.

29. Dejour H, Selmi TAS, Dejour D, Neyret Ph. Resultats a plus des 10 ans de 52 laxites antérieures isolées tratées par greffe libre de tendon rotulien associée à une plastie de Lemaire. Rev Cir Orthop Reparatrice Appar Mot. 1996;82 Suppl 2:83-4. [71st annual meeting of the French Society of Surgical Orthopedics and Traumatology. Abstract].
30. McGuire DA, Wolchok JC. Extra-articular lateral reconstruction technique. Arthroscopy. 2000;16(5):553-7.

31. Noyes FR, Barber-Westin SD. Revision anterior cruciate surgery with use of bone-patellar tendon-bone autogenous grafts.J Bone Joint Surg Am. 2001;83-A(8):1131-43.

32. Amatuzzi MM. A cirurgia do joelho no Brasil. In: Amatuzzi MM. Joelho. São Paulo: Editora Roca; 2004. cap. 54, p. 470-8.

33. Fu FH, Zelle BA. Rotational instability of the knee: editorial comment Clin Orthop Relat Res. 2007;454:3-4.

34. Casteleyn PP. Management of anterior cruciate ligament lesions: surgical fashion, personal whim or scientific evidence? Study of medium- and long-term results. Acta Orthop Belg. 1999;65(3):327-39.

35. Diekstall P, Rauhut F. [Considerations for the indications for anterior cruciate ligament reconstruction. Results of conservative versus operative treatment]. Unfallchirurg. 1999;102(3):173-81. German.

36. Renström PA. [Anterior cruciate ligament injuries - still an enormous challenge. Life style-related surgery likely to be used more widely]. Lakartidningen. 1999;96(19):2349-52, 2354, 2356. Swedish.

37. Reer R, Nagel V, Paul B, Edelmann H, Braumann KM. [The application of external knee stabilizers - Influence on mechanical stabilization and physical performance]. Sportverletz Sportschaden. 2001;15(3):62-7. German.

38. Fujimoto E, Sumen Y, Ochi M, Ikuta Y. Spontaneous healing of acute anterior cruciate ligament (ACL) injuries - conservative treatment using an extension block soft brace without anterior stabilization. Arch Orthop Trauma Surg. 2002;122(4):212-6.

39. Hinterwimmer S, Engelschalk M, Sauerland S, Eitel F, Mutschler W [Operative or conservative treatment of anterior cruciate ligament rupture: a systematic review of the literature]. Unfallchirurg. 2003; 106(5):374-9. German.

40. Crain EH, Fithian DC, Paxton EW, Luetzow WF. Variation in anterior cruciate ligament scar pattern: does the scar pattern affect anterior laxity in anterior cruciate ligament-deficient knees? Arthroscopy. 2005;21(1): 19-24.

41. Linko E, Harilainen A, Malmivaara A, Seitsalo S. Surgical versus conservative interventions for anterior cruciate ligament ruptures in adults. Cochrane Database Syst Rev. 2005;(2):CD001356. Update of: Brain Res Dev Brain Res. 1994;78(2):226-36.

42. Fitzgerald GK, Axe MJ, Snyder-Mackler L. A decision-making scheme for returning patients to high-level activity with nonoperative treatment after anterior cruciate ligament rupture. Knee Surg Sports Traumatol Arthrosc. 2000;8(2):76-82.

43. Strehl A, Eggli S. The value of conservative treatment in ruptures of the anterior cruciate ligament (ACL). J Trauma. 2007;62(5):1159-62. 